\title{
Quelques annotations sur le théâtre de Luis Araújo et apostilles à Libre échange
}

Isabelle Reck

\section{(2) OpenEdition}

1 Journals

Édition électronique

URL : https://journals.openedition.org/cher/416

DOI : 10.4000/cher.416

ISSN : 2803-5992

Éditeur

Presses universitaires de Strasbourg

Édition imprimée

Date de publication : 19 novembre 2020

Pagination : 203-208

ISBN : 979-10-344-0071-3

ISSN : 1968-035X

\section{Référence électronique}

Isabelle Reck, "Quelques annotations sur le théâtre de Luis Araújo et apostilles à Libre échange », reCHERches [En ligne], 25 | 2020, mis en ligne le 01 octobre 2021, consulté le 19 novembre 2021. URL : http://journals.openedition.org/cher/416 ; DOI : https://doi.org/10.4000/cher.416 


\title{
Quelques annotations sur le théâtre de Luis Araújo et apostilles à Libre échange
}

\author{
ISABELLE RECK
}

\section{Brève présentation du théâtre de Luis Araújo}

Luis Araújo, homme de théâtre complet, a exploré tous les métiers des arts de la scène - auteur, metteur en scène, scénariste, acteur, directeur de compagnie -, avant de se consacrer plus exclusivement à l'écriture théâtrale: on lui doit une vingtaine de textes dramatiques depuis sa première pièce écrite en 1983. Son théâtre est avant tout un théâtre engagé, politique et à forte portée éthique et sociale, qui explore les grandes questions de notre époque: les droits de l'Homme, la fascination du pouvoir et les violence qu'elle engendre, la servitude volontaire, l'impact du système économique et financier sur nos vies, l'écologie, notre responsabilité envers les générations futures, les crises migratoires, la biotechnologie, les mutants cybernétiques et l'eugénisme.

Il a recréé des événements du passé et puisé des éléments dans des biographies marquantes - Vanzetti, Kafka enamorado - pour mieux parler de ces problématiques au présent, "[n'installant] pas l'Histoire sur la scène, mais [situant] la scène et la salle dans l'Histoire ${ }^{1}$. D'autres pièces, au contraire, comme Fantastic calentito ou Prototipo de poniente, ont choisi un genre peu fréquent au théâtre, la sciencefiction. Pour Luis Araújo, ce genre est le plus à même de parler vraiment de la réalité, de la penser, de créer l'espace d'une utopie ou d'une dystopie et de poser les questions qui relèvent de la philosophie de l'esprit et de la philosophie politique. Dans «Nota del autor» à sa pièce Prototipo de poniente, inspirée précisément d'un récit de Stanislaw Lem, il manifeste son admiration pour des auteurs de science-fiction tels que Ray Bradbury, Stanislaw Lem, Phillip K. Dick, Adlous Huxley, Theodore Sturgeon, Ursula K. Le Guin. Ce sont, pour lui, les «vrais penseurs qui nous obligent à nous poser les vraies questions importantes» :

1 Bernard Dort, Théâtres essais, Paris, editions du seuil, Points, 1986, p. 243. 
[...] ces auteurs [...] posent vraiment la question du type de société que nous sommes en train de construire, que nous souhaitons léguer au futur. Ils interrogent: jusqu'à quel point sommes nous conscients de la responsabilité que nous avons au regard de la société et de l'espèce humaine en général. [...] notre société actuelle s'oriente dans une voie qui fait de nous des prédateurs [...] ce sont les vrais penseurs qui nous obligent à nous poser les vraies questions importantes².

Luis Araújo a mis en exergue à sa pièce La construcción de la catedral une citation d'Antonio Buero Vallejo: «Nous avons besoin d'un réarmement moral de notre société, et que l'honnêteté y redevienne une valeur ${ }^{3}$.» Son théâtre s'investit en effet avant tout d'une fonction éthique: il se doit de développer une "éthique de la responsabilité", une éthique de l'action pour l'avenir. Il est surtout un théâtre de "l'éthique du futur", que ces mots de Vladimir Jankélévitch résument bien: "Peut-on se souvenir quand on n'anticipe jamais? Qui se souvient anticipera un jour ${ }^{4}$."

Le théâtre de Luis Araújo, théâtre ontologique, philosophique, politique et éthique, n'a de cesse de se poser la question même du théâtre et de l'art en général dans nos societés, leur place, leurs fonctions, leur mission, leur impact. Deux des pièces abordent plus frontalement cette question Carmen Privatta et La Parte contratante.

Si son théâtre n'a pas été mis en scène à la hauteur de ce qu'il mérite, deux de ses pièces ont été montées dans des théâtres prestigieux de Madrid, Teatro Español et Teatro María Guerrero du Centro Dramático Nacional, respectivement: Mercado libre en 2009 (mise en scène de Jesús Cracio) et Kafka enamorado en 2013 (mise en scène de José Pascual).

\section{Libre échange de Luis Araújo: Une parabole de la marchandisation des relations humaines}

Libre échange 5 , Mercado libre dans sa version originale espagnole, a remporté le prix Esperpento 2008, qui récompense les meilleurs textes dramatiques. Mis en scène par Jesús Cracio pour le Teatro Español de Madrid, la première a eu lieu dans la petite salle de ce théâtre en décembre 2009.

Libre échange, le titre le dit d'emblée, la pièce est une parabole de la manière dont le système économique et financier organise nos vies et dont l'idéologie économiciste pèse sur les relations humaines.

2 «Entrevista con Luis Araujo», dans El juego eterno. Teatro de Luis Araújo, éd. de John P. Gabriele, Madrid, Editorial Fundamentos, «Espiral/teatro», p. 219. La traduction est de nous.

3 «Habría que conseguir un rearme moral, que la honestidad volviera a ser un valor en nuestra sociedad».

4 Vladimir Jankélévitch, L'irreversible et la nostalgie, Paris, Flammarion, Nouvelle bibliothèque scientifique dirigée par Fernand Braudel, 1974, p. 51.

5 Araújo L., Mercado libre, Madrid, Cuadernos del teatroespañol, 2009. 
Au tournant de ce $\mathrm{xxI}^{\mathrm{e}}$ siècle, la crise des subprimes de 2007-2008 qui a laissé exsangues tant de pays, notamment l'Espagne, et ruiné et anéanti tant de vies, a donné la mesure des ravages de ces Gorgones aux «faces répugnantes » (Ovide). La pièce est précisément écrite en 2007 et représentée en 2009. Elle s'inscrit dans l'orientation forte que prend le théâtre espagnol à partir de cet événement majeur, la «dramaturgie de la crise», voire elle l'inaugure. C’est « une pièce rapide», née, dans ce contexte, comme une urgence, comme l'explique Luis Araújo: «J'ai mis des années à écrire certains de mes textes», «je peux facilement ruminer une réplique pendant trois jours », mais Libre échange, «je l'ai pensé et écrit en à peine cinq mois. ${ }^{6}{ }$

«J'ai toujours été pour un théâtre immédiat», déclare Jesús Cracio (2009: $130)$, le metteur en scène, pour «des pièces qu'on ne doit pas laisser sur les rayons d'une bibliothèque à prendre la poussière», car elles «nous rappellent les valeurs de cet enfer consumériste et médiatique qui est en train de nous dévorer ", et servent de "miroir-reflet de nos vies. " Libre échange est un théâtre immédiat, un théâtre d'urgence, engagé. Il est, selon la métaphore de Jesús $\mathrm{Cracio}^{7}$, «la lame d'un couteau aiguisé.»

Alain Eraly, dans son livre Quand les mots construisent la réalité. La performatibilité de l'économie, décrit les différents modes de performation, c'est à dire «les processus par lesquels une théorie peut agir sur le monde social» et «les médiations sociales par lesquelles une théorie en vient à se glisser dans des rapports contraignants et à affecter la vie des gens qui, pour l'essentiel, ignorent tout de cette théorie», en l'occurrence la théorie économique comme représentation du monde. Si le personnage $B$, immigrée latino-américaine en situation irrégulière, mère de trois enfants et prostituée, est la victime de ce processus et le dénonce, il n'en ai pas de même de A, riche avocat cynique, qui ne voit le monde et les relations que sous l'angle économique de l'offre et la demande, de l'achat/vente, et les met au service de la réalisation de son «désir». Luis Araújo joue en permanence de la polysémie du verbe espagnol «querer» (aimer, vouloir) difficile à rendre dans la traduction française, pour esquisser les frontières entre le besoin, la nécessité, le désir et le caprice, entre désirer et vouloir dominer: "A.-Ne puis-je pas faire ce que je veux avec ce qui est à moi ?». La prostituée vend son corps pour survivre, l'avocat blasé qui l'achète ne s'en contente pas. Elle a besoin de l'avocat pour aider ses fils adolescents accusés - ironie du destin? - du viol d'une camarade de classe. Il veut ce qui n'est pas à vendre.

6 Araújo, L., «Escribir en el mercado», dans Luis Araújo, Mercado libre, Madrid, Cuadernos del teatroespañol, 2009, p. 133-142, ici p. 134: «Me puedo pasar fácilmente tres días rumiando una réplica [...] Algunos de mis textos he tardado años en escribirlos». "Para mí es una obra rápida. La pensé y la escribí en apenas cinco meses.»

7 Cracio, J., «Por un teatro inmediato», dans Luis Araújo, Mercado libre, Madrid, Cuadernos del teatroespañol, 2009, p. 119-132, ici p. 123. 
Luis Araújo met ainsi en scène la marchandisation des rapports amoureux sur le mode sado-masochiste de la servitude volontaire. Le dialogue déploie toute la violence de ces rapports, de ce corps à corps. Dans la mise en scène de Cracio, les acteurs sont nus sur un immense lit, plate-forme de forme circulaire: «je vois un ring où deux âmes se donnent des coups de poings. Deux acteurs qui s'exposent et dénudent leurs âmes plus que leur corps.» Il lui fallait amener les spectateurs à ne plus voir cette nudité, dérangeante pour certains, mais à la leur faire ressentir comme «les entrailles de ces âmes mises à nue ${ }^{8}$.

Le dialogue est ponctué, au début, de petits mots d'amour: "mon amour», "mon cœur». Dans les premières scènes, B, qui a déjà connu beaucoup d'épreuves, décrit le monde sous l'aspect sombre de la loi de la jungle. À partir de la scène IV, le cynique avocat va lui en offrir le pire exemple. Les tortures qu'il lui impose vont en s'amplifiant. Il n'est jamais rassasié, il veut toujours plus. Sa violence de prédateur explose dans le dénouement.

Voici un petit florilège de répliques qui illustrent comment la théorie économique imprègne et détermine la représentation du monde»: «Toi, combien tu coûtes?", "Je veux que tu sois à moi», "Moi, j'achète ce qui est mis en vente.», "Je donnerais n'importe quoi pour te posséder ainsi toujours.», "Combien crois-tu que vaut ta vie?». B décrit bien ce processus de «performation» dans l'une de ses réponses: «Tu pourrais faire de moi ce que tu veux... mais tu sais très bien que... ce n'est pas ce que tu veux... Le monde n'est pas comme tu le décris... tu as tort... les gens ne sont pas... C'est toi qui fais $\mathrm{du}$ monde ce qu'il est.»

\section{Notice bio-bibliographique de Luis Araújo}

Luis Araújo se consacre actuellement principalement à l'écriture. On lui doit une vingtaine de textes dramatiques. Cependant, il a exercé presque tous les métiers des arts de la scène: auteur, metteur en scène, scénariste, acteur, directeur de compagnie, critique de théâtre.

Luis Araújo s'est formé à la mise en scène à la R.E.S.A.D. (Madrid). Il est également titulaire d'une licence en études hispaniques (Université Complutense de Madrid) et d'un Master ès arts (Montréal, Canada) et diplômé dans le domaine de l'audiovisuel (Redacción y locución audiovisual) et de la Coopération internationale pour le développement (Instituto de Empleo de la Comunidad Autónoma de Madrid).

Il a collaboré, à Paris, avec les compagnies Jean-Louis Barrault-Madeline Renaud et Pierre Chabert, et, à Madrid, entre autres, avec Tábano, Teatro Libre, Pícaro Teatro, Artefacto, et dirigé les compagnies: Tres tristes libres, C.C.C.K. et Culebrón portátil. Il a mis en scène plusieurs de ses pièces et des pièces d'autres auteurs. Il a

8 Cracio, J., "Por un teatro inmediato», dans Luis Araújo, Mercado libre, Madrid, Cuadernos del teatroespañol, 2009, p. 119-132, ici p. 123 et 130. 
réalisé à Madrid une cinquantaine de lectures dramatiques (semimontados) d'auteurs contemporains dans des théâtres ou des institutions comme Centro Dramático Nacional, Teatro Español, Teatro Real, Círculo de Bellas Artes, Centro Cultural de la Villa de Madrid, Ateneo de Madrid, et dans différentes salles privées et alternatives.

Luis Araújo a exercé aussi en tant qu'enseignant. Il a ainsi animé de nombreux ateliers de formation à l'écriture dramatique à la Escuela de Letras, au Centro Nacional de Nuevas Tendencias Escénicas, à la R.E.S.A.D., à la Fundación Autor (Madrid), au I.S.A.D.A.C. de Rabat (Maroc). Il a été professeur d'interprétation à l'Université de Montreal (Canada) et à l'Instituto de Estudios Europeos de Madrid. Il a assuré en outre des cours sur le théâtre du Siècle d'Or, le théâtre élisabéthain et des cours de mise en scène à l'University of Kent at Canterbury. Il a animé des séminaires de traduction théâtrale au centre Européen de Traduction littéraire de Bruxelles et des séminaires de Master sur le théâtre et la pratique théâtrale à l'Université de Strasbourg (2014-2017). Il a été, par ailleurs, secrétaire général (1992-1995) et vice-président (1995-1998) de la Asociación de Autores de Teatro de España (AAT) et a été assesseur Expert dans le domaine de la Culture de la E.A.C.E.A. (Comission Européenne).

Enfin, très actif dans le domaine de la critique et de la théorie théâtrales, il a à son actif de très nombreuses études et articles dans des revues spécialisées comme Primer Acto.

\section{Pièces de théâtre de Luis Araújo, par ordre chronologique de leur publication}

Carlota o La noche mejicana (version espagnole de l'original de Liliane Wouters) dans Tramoya, no 37, oct-dic. 1993, Universidad de Veracruz (México).

Vanzetti, dans Primer acto, no 254, mars 1994 (Madrid); dans Madrid, Ed. Visor, col. «Biblioteca Antonio Machado de Autores Contemporáneos», 1996; dans Contemporary Spanish Plays, New Brunswick (New Jersey), Ed. Estreno, 1999 (dans sa traduction anglaise).

Carmen Privatta, dans Monólogos I, Madrid, Ediciones Asociación de Autores de Teatro, col. «Teatro Asociado», 1994.

Las aventuras $y$ andanzas del Aurelio y la Constanza (finaliste du IV Premio Iberoamericano de Dramaturgia Infantil, 1995), dans IV Premio Iberoamericano de Dramaturgia Infantil. Obras galardonadas, Bilbao, Ediciones del Centro de Documentación de Títeres de Bilbao, 1997.

La parte contratante, dans Escena, no 29, Abril 1996 (Barcelona).

Luna negra dans Teatro urgente, Madrid, Editorial La Avispa, col. «El ojo de la avispa», 1997 (avec Fantastic calentito); dans Estreno, vol. XXIII, n² 2, Automne 1997, Delaware, Ohio (USA); dans Tramoya, n 55, avril-juin 1998, Universidad de Veracruz (México); dans Black moon, Milikin University, Decatur, Illinois (USA), 2005.

Fantastic calentito, dans Teatro urgente, Madrid, Editorial La Avispa, Col. «El ojo de la Avispa", 1997 (avec Luna negra); dans Tramoya, no 74, janvier/mars 2003, Universidad de Veracruz (México). 
Los gatos blancos, Al borde del área, Alicante, Muestra de Teatro español de autores contemporáneos, col. "Teatro español contemporáneo », 1998; dans Monólogos escénicos, Madrid, Ediciones Federación de Artistas del Estado Español, 2010.

La construcción de la catedral (Prix internacional Tramoya du meilleur texte dramatique en langue espagnole, 2000), dans Tramoya, no 65, oct.-déc. 2000, Universidad de Veracruz (México); dans El Juego Eterno. Teatro de Luis Araújo, éd. De J. Gabriele, Madrid, Ed. Fundamentos, col. «Espiral/teatro», 2001 (pièce publiée avec Prototipo de poniente); dans Teatro spagnolo contemporaneo. I giovani drammaturghi, Alessandria, Edizionni de l'Orso, vol. III. Emilio Coco, 2004.

Prototipo de poniente dans El Juego Eterno: Teatro de Luis Araújo, édition de J. Gabriele, Madrid, Ed. Fundamentos, col. «Espiral/teatro», 2001 (pièce publiée avec La construcción de la catedral).

Trenes que van al mar, dans Madrid, Ediciones Asociación de Autores de Teatro/ Comunidad de Madrid, col. «Damos la Palabra», 2001; dans Tramoya, n 78, janvier-mars, 2004, Universidad de Veracruz (México).

Enemigo, dans Diagonal, n 12, 1-14 septembre, 2005 (Madrid); dans El tamaño no importa. Textos breves de aquí y de ahora, Madrid, Asociación de Autores de Teatro, "Teatro", no 18, 2011.

Sin novedad dans Diagonal, no 31 y 32, 25 mai-7 juin, 2006 (Madrid).

Las palabras y los hechos dans Maratón de monólogos, 2007, Madrid, Ed. Asociación de Autores de Teatro, 2008.

Mercado libre (Prix Esperpento 2008), dans Artez, octobre 2008 (Bilbao); Madrid. Ed. A.A.T./ C.A.M., col. «Damos la palabra», 2008; Madrid, Teatro Español, 2009; et une version grecque: Eleutheri agora, Athènes, Ed Aparsis, 2012.

Trayectoria de la bala (Prix international de dramaturgie Fundación Cultura Frontal, Buenos Aires); dans Teatro de Papel, no 12, Madrid, Ed. Primer Acto, 2010; dans Premio Teatro Incluido, Ciudad autónoma de Buenos Aires, Ed. Ópera Prima/ Fundación Cultura Frontal, 2010.

¿Tú me querías?, dans Maratón de monólogos, Madrid, Asociación de Autores de Teatro, 2010.

Por que as acacias non han dar xarope?, dans Revista Galega de Teatro, n 64. Textos/61, automne 2010.

Kafka enamorado, dans Autores en el Centro, no 8, Madrid, Centro Dramático Nacional. INAEM, Ministerio de Educación Cultura y Deporte, 2013.

Dios está muy lejos, dans Acotaciones, janvier-juin 2014 (Madrid, RESAD), p. $79-132$.

No perdáis este tren, dans Tres comedias del miedo, édition de Francisco Gutiérrez Carbajo, Madrid, Ed. Cátedra, 2016, p. 203-230. 